\title{
Genetic structure of Salmonella revealed by fragment analysis
}

Molecular Biology Unit, Virus Reference Division ${ }^{1}$ and Laboratory of Enteric Pathogens ${ }^{2}$, Central Public Health Laboratory, 61 Colindale Avenue, London NW9 5HT, UK

\author{
Fiona Scott, ${ }^{1}$ John Threlfall ${ }^{2}$ and Catherine Arnold ${ }^{1}$
}

Author for correspondence: Catherine Arnold. Tel: +44 2082004400 . Fax: +44 2082001569.
e-mail: carnold@phls.nhs.uk

Keywords: FAFLP, Salmonella, fragment analysis

\section{INTRODUCTION}

Salmonella are routinely classified by serotype on the basis of expression of three surface antigens $(\mathrm{Ag})$, the somatic $\mathrm{O} \mathrm{Ag}$, the flagella $\mathrm{H} 1$ and $\mathrm{H} 2 \mathrm{Ags}$ and the capsular Vi Ag, according to the Kauffmann-White scheme. This scheme recognizes in excess of 2400 serotypes (Rowe \& Hall, 1989). The majority of serotypes, including those responsible for disease in humans, are found in Salmonella choleraesuis (commonly known as Salmonella enterica) subspecies I (Beltran et al., 1988). A number of subtyping schemes have been described for the most common and medically most important serotypes, including phage typing (Ward et al., 1987; Briggs \& Fratamico, 1999; Kariuki et al., 1999b), pulsed-field gel electrophoresis (Powell et al., 1994; Thong et al., 1995; Ridley et al., 1996; Kariuki et al., 1999a, b; Laconcha et al., 2000),

Published online ahead of print on 19 April 2002 as DOI 10.1099/ ijs.0.02100-0.

Versions of the trees in Figs 2-4 showing various serotypes identified in colour are available as supplementary material in IJSEM Online (http://ijs.sgmjournals.org/).

Abbreviations: ET, electrophoretic type; FAFLP, fluorescent amplifiedfragment length polymorphism; MLEE, multilocus enzyme electrophoresis; MLST, multilocus sequence typing. plasmid profile analysis (Threlfall et al., 1989, 1994; Ridley et al., 1998; Kariuki et al., 1999a), ribotyping (Olsen et al., 1997; Ridley et al., 1998; Old et al., 1999, 2000) and IS200 fingerprinting (Stanley et al., 1991, 1992; Ezquerra et al., 1993; Olsen et al., 1994, 1997; Pelkonen et al., 1994; Weide-Botjes et al., 1998; Old et al., 1999, 2000; Millemann et al., 2000). However, neither these methods nor serotyping provides an adequate basis for determining the overall levels of genetic relatedness between strains and they therefore have no application in population genetics (Selander $e t$ al., 1986; Beltran et al., 1988).

Multi-locus enzyme electrophoresis (MLEE) has been used to measure genetic variation within bacterial populations, and the degree of overall genetic relatedness among isolates, populations and species, of a number of genera including Escherichia, Salmonella, Legionella, Neisseria and Haemophilus (Selander \& Musser, 1990). MLEE analysis characterizes isolates on the basis of the relative electrophoretic mobilities of various water-soluble cellular enzymes. As the net electrostatic charge (and therefore the rate of migration) of a protein during electrophoresis is determined by its amino acid sequence, the mobility variants (electromorphs) of an enzyme can be equated directly with alleles at a corresponding structural gene locus (Selander et al., 1986). 
MLEE studies of populations of Salmonella have described their basically clonal structure, with a few predominant clones achieving widespread if not global distribution (Selander et al., 1986; Beltran et al., 1988, 1991; Boyd et al., 1993). Three Salmonella reference collections (SARA, SARB and SARC), which include 160 Salmonella strains, have been chosen by MLEE to represent the scope of genetic diversity within natural populations of Salmonella. These collections include representatives of the Salmonella species $S$. choleraesuis, Salmonella bongori, Salmonella typhi, Salmonella enteritidis and Salmonella typhimurium and the six subspecies of S. choleraesuis (Beltran et al., 1991; Boyd et al., 1993, 1996).

For a number of organisms, such as Neisseria meningitidis (Maiden et al., 1998), Streptococcus pneumoniae (Enright \& Spratt, 1998) and Staphylococcus aureus (Enright et al., 2000), MLEE analysis is now being superseded by multilocus sequence typing (MLST), the partial sequencing of some of the housekeeping genes analysed in MLEE. Although sequencing of a few individual genes of Salmonella has also been described, including the isocitrate dehydrogenase gene icd (Wang et al., 1997), the 6-phosophogluconate hydrogenase gene gnd (Nelson \& Selander 1994), the malate dehydrogenase gene $m d h$ (Boyd et al., 1994) and the invasion genes (Li et al., 1995; Boyd et al., 1996), this multilocus sequencing approach has yet to be fully evaluated and therefore adopted for Salmonella.

Fluorescent amplified-fragment length polymorphism (FAFLP) is an accurate, rapid and reproducible method that permits simultaneous sampling of multiple loci throughout the bacterial genome (Arnold et al., 1999a). It is a flexible technique where the degree of resolution can easily be modified by choice of primers, thereby allowing a range of applications from highresolution genotyping for outbreak recognition to lower-resolution population structure investigations. AFLP has been used in studies of the higher eukaryotes to determine the genetic structure of populations (Sanchez et al., 1999; Katiyar et al., 2000; Keiper \& McConchie, 2000) and, for prokaryotes, FAFLP data have correlated well with those generated by MLEE, e.g. for the EcoR collection of Escherichia coli (Ochman \& Selander, 1984; Arnold et al., 1999b).

Five species are recognised in classical Salmonella taxonomy: S. choleraesuis, S. bongori, S. typhimurium, $S$. enteritidis and S. typhi. S. choleraesuis is further divided into six subspecies: I, choleraesuis; II, salamae; IIIa, arizonae; IIIb, diarizonae; IV, hautenae; and VI, indica (Beltran et al., 1988; Uzzau et al., 2000).

Medical microbiology uses a different taxonomic system for Salmonella, based on MLEE, DNA hybridization and sequencing studies. There are two recognized species of the genus Salmonella: S. choleraesuis (commonly known as $S$. enterica) and $S$. bongori (Uzzau et al., 2000). S. choleraesuis is again divided into six subspecies. S. bongori was previously con- sidered to be subspecies V of S. choleraesuis; however, MLEE and DNA hybridization studies have revealed that it is sufficiently different from the other subspecies to be recognised as a separate species (Reeves et al., 1989). The most recent studies have further subdivided subspecies IV into subspecies IV and VII (Boyd et al., 1996). For the purposes of this paper, the classical Salmonella taxonomic nomenclature has been used and, where serotype names are used (shown with a capital letter and not italicized, e.g. S. Paratyphi B), these refer to serotypes of $S$. choleraesuis subspecies I.

In this study, we have used FAFLP to determine the genetic structure of the salmonellae as represented by the three Salmonella reference collections.

\section{METHODS}

Bacterial strains and growth conditions. Salmonella reference collections A, B and C (Table 1) were received from the Salmonella Genetic Stock Centre, University of Calgary, Canada. These strains have been characterized by MLEE; the strain designations used in this study are the same as in the original papers (Beltran et al., 1991; Boyd et al., 1993, 1996). Collections SARA and SARB consist entirely of $S$. choleraesuis subspecies I. SARA is composed of 72 strains of five serotypes representing the $S$. typhimurium clonal complex of $S$. choleraesuis (Beltran et al., 1991) and SARB strains represent the genomic variation among the common serotypes of subspecies I. SARB consists of 72 strains of 37 serotypes including 18 serotypes represented by one electrophoretic type (ET) and 19 serotypes of multiple electrophoretic types, eight of which were apparently monophyletic (i.e. thought to be derived from a recent common ancestor through mutational divergence). The ETs of these eight serotypes were genotypically similar by MLEE, while the other 11 were polyphyletic (Boyd et al., 1993). SARC consists of two representative strains of $S$. bongori and each of the seven Salmonella subspecies recognised by MLEE (Boyd et al., 1996).

All strains were cultured aerobically on nutrient agar plates for $16 \mathrm{~h}$. Stock cultures were stored on Protect bacterial preservers (Technical Service Consultants) at $-70^{\circ} \mathrm{C}$.

FAFLP. Genomic DNA was extracted by using the Qiagen DNeasy tissue kit. DNA was eluted into a final volume of $50 \mu \mathrm{l}$ distilled water. FAFLP was performed as described by Desai et al. (1998). Briefly, MseI and EcoRI were used to digest approximately $500 \mathrm{ng}$ genomic DNA for $2 \mathrm{~h}$. To the digested DNA was added 5.0 $\mu 110 \times$ T4 DNA ligase buffer, 400 U T4 DNA ligase (New England Biolabs), $16 \cdot 8 \mu 1$ sterile distilled water and final concentrations of $1 \mathrm{nM} E c o+0$ adapter (5'-CTCGTAGACTGCGTACC-3' and 3'-CTGACGCATGGTTAA-5') and $5 \mathrm{nM}$ Mse adapter (5'-GACGATGAGTCCTGAG-3' and 3'-CTACTCAGGACTCAT-5') (both from Sigma-Genosys), to give a final reaction volume of $50 \mu \mathrm{l}$. Decreased adapter concentrations were used to reduce the production of $n+3$ FAFLP fragments (data not shown).

PCR was performed in a total reaction volume of $25 \mu \mathrm{l}$ consisting of $2.5 \mu \mathrm{l}$ of the above digestion/ligation reaction, $2.5 \mu \mathrm{l} 10 \times$ PCR buffer, $2.5 \mathrm{mM} \mathrm{MgCl}_{2}, 0.2 \mathrm{mM}$ dNTPs (all from Life Technologies), $0 \cdot 2 \mu \mathrm{M}$ 6-carboxyfluorescein (6FAM) fluorescently labelled $E c o+0$ primer (5'-6-FAMGACTGCGTACCAATTC-3'), $1 \mu \mathrm{M} M s e+$ TA primer (5'-GATGAGTCCGAGTAATA-3') (both primers from 
Table 1. SARA, SARB and SARC strains

SARA consists of 72 strains of the $S$. typhimurium complex (Beltran et al., 1991), SARB consists of 72 strains of the common serotypes of $S$. choleraesuis (Boyd et al., 1993) and SARC consists of 16 strains representing the $S$. choleraesuis subspecies and $S$. bongori (Boyd et al., 1996).

\begin{tabular}{|c|c|c|c|c|c|c|c|}
\hline Strain & Subsp. & Serotype & Antigenic formula & ET & Biotype & Locality & Source \\
\hline \multicolumn{8}{|l|}{ SARA } \\
\hline A01 & I & S. typhimurium & $1,4,[5], 12: \mathrm{i}: 1,2$ & $\operatorname{Tm} 1$ & $1 \mathrm{f}$ & Mexico & Human \\
\hline $\mathrm{A} 02$ & I & S. typhimurium & $1,4,[5], 12: \mathrm{i}: 1,2$ & $\operatorname{Tm} 1$ & & Lab strain & \\
\hline $\mathrm{A} 03$ & I & S. typhimurium & $1,4,[5], 12: \mathrm{i}: 1,2$ & $\operatorname{Tm} 1$ & $11 \mathrm{di}$ & Rhode Island, USA & Horse \\
\hline A04 & I & S. typhimurium & $1,4,[5], 12: \mathrm{i}: 1,2$ & $\operatorname{Tm} 1$ & $25 \mathrm{e}$ & Indiana, USA & Rabbit \\
\hline $\mathrm{A} 05$ & I & S. typhimurium & $1,4,[5], 12: \mathrm{i}: 1,2$ & $\operatorname{Tm} 1$ & $9 \mathrm{fi}$ & Mongolia & \\
\hline A06 & I & S. typhimurium & $1,4,[5], 12: \mathrm{i}: 1,2$ & $\operatorname{Tm} 2$ & $17 \mathrm{a}$ & Ohio, USA & Human \\
\hline A07 & I & S. typhimurium & $1,4,[5], 12: \mathrm{i}: 1,2$ & $\operatorname{Tm} 3$ & $1 \mathrm{a}$ & Norway & \\
\hline A08 & I & S. typhimurium & $1,4,[5], 12: \mathrm{i}: 1,2$ & $\operatorname{Tm} 5$ & $1 \mathrm{a}$ & Finland & \\
\hline A09 & I & S. typhimurium & $1,4,[5], 12: \mathrm{i}: 1,2$ & $\operatorname{Tm} 7$ & $1 \mathrm{a}$ & California, USA & Parrot \\
\hline $\mathrm{A} 10$ & I & S. typhimurium & $1,4,[5], 12: \mathrm{i}: 1,2$ & $\operatorname{Tm} 9$ & $1 \mathrm{~b}$ & California, USA & Opossum \\
\hline A11 & I & S. typhimurium & $1,4,[5], 12: \mathrm{i}: 1,2$ & $\operatorname{Tm} 10$ & $2 \mathrm{a}$ & Thailand & \\
\hline $\mathrm{A} 12$ & I & S. typhimurium & $1,4,[5], 12: \mathrm{i}: 1,2$ & Tm 11 & 32 begi & Louisiana, USA & Horse \\
\hline A13 & I & S. typhimurium & $1,4,[5], 12: \mathrm{i}: 1,2$ & Tm 12 & $29 \mathrm{~b}$ & France & \\
\hline A14 & I & S. typhimurium & $1,4,[5], 12: \mathrm{i}: 1,2$ & $\operatorname{Tm} 13$ & $1 \mathrm{a}$ & Panama & \\
\hline A 15 & I & S. typhimurium & $1,4,[5], 12: \mathrm{i}: 1,2$ & $\operatorname{Tm} 14$ & $17 \mathrm{a}$ & Texas, USA & Dog \\
\hline A16 & I & S. typhimurium & $1,4,[5], 12: \mathrm{i}: 1,2$ & $\operatorname{Tm} 15$ & $3 \mathrm{~d}$ & N. Carolina, USA & Human \\
\hline A17 & I & S. typhimurium & $1,4,[5], 12: \mathrm{i}: 1,2$ & $\operatorname{Tm} 16$ & $3 \mathrm{~d}$ & Yugoslavia & \\
\hline A18 & I & S. typhimurium & $1,4,[5], 12: \mathrm{i}: 1,2$ & Tm 17 & $3 \mathrm{~d}$ & Iowa, USA & Horse \\
\hline A19 & I & S. typhimurium & $1,4,[5], 12: \mathrm{i}: 1,2$ & $\operatorname{Tm} 21$ & $1 \mathrm{f}$ & Mexico & Human \\
\hline $\mathrm{A} 20$ & I & S. typhimurium & $1,4,[5], 12: \mathrm{i}: 1,2$ & $\operatorname{Tm} 22$ & $1 \mathrm{a}$ & France & \\
\hline A21 & I & S. typhimurium & $1,4,[5], 12: \mathrm{i}: 1,2$ & $\operatorname{Tm} 23$ & & Oregon, USA & Heron \\
\hline $\mathrm{A} 22$ & I & $S$. Saintpaul & $1,4,[5], 12: e, h: 1,2$ & $\mathrm{Sp} 1$ & & Massachusetts, USA & Human \\
\hline $\mathrm{A} 23$ & I & $S$. Saintpaul & $1,4,[5], 12: e, h: 1,2$ & $\mathrm{Sp} 2$ & & Pennsylvania, USA & Human \\
\hline A24 & I & $S$. Saintpaul & $1,4,[5], 12: e, h: 1,2$ & Sp 3 & & Texas, USA & Human \\
\hline A25 & I & $S$. Saintpaul & $1,4,[5], 12: e, h: 1,2$ & Sp 3 & & France & \\
\hline A26 & I & S. Saintpaul & $1,4,[5], 12: e, h: 1,2$ & Sp 3 & & France & Human \\
\hline A27 & I & $S$. Saintpaul & $1,4,[5], 12: e, h: 1,2$ & Sp 3 & & France & Human \\
\hline A28 & I & $S$. Saintpaul & $1,4,[5], 12: \mathrm{e}, \mathrm{h}: 1,2$ & Sp 3 & & France & Human \\
\hline A 29 & I & $S$. Saintpaul & $1,4,[5], 12: e, h: 1,2$ & Sp 4 & & Florida, USA & Human \\
\hline $\mathrm{A} 30$ & I & $S$. Heidelberg & $1,4,[5], 12: \mathrm{r}: 1,2$ & $\mathrm{He} 1$ & $9 \mathrm{i}$ & Pennsylvania, USA & Chicken \\
\hline A31 & I & $S$. Heidelberg & $1,4,[5], 12: r: 1,2$ & $\mathrm{He} 1$ & $9 \mathrm{i}$ & Maryland, USA & Swine \\
\hline A32 & I & $S$. Heidelberg & $1,4,[5], 12: r: 1,2$ & $\mathrm{He} 1$ & $9 \mathrm{i}$ & Texas, USA & Dog \\
\hline A33 & I & $S$. Heidelberg & $1,4,[5], 12: r: 1,2$ & $\mathrm{He} 1$ & $9 \mathrm{gi}$ & Mexico & Human \\
\hline A 34 & I & $S$. Heidelberg & $1,4,[5], 12: \mathrm{r}: 1,2$ & $\mathrm{He} 1$ & $9 \mathrm{i}$ & Israel & \\
\hline A 35 & I & $S$. Heidelberg & $1,4,[5], 12: r: 1,2$ & $\mathrm{He} 2$ & $9 \mathrm{i}$ & Brazil & \\
\hline A36 & I & $S$. Heidelberg & $1,4,[5], 12: r: 1,2$ & $\mathrm{He} 3$ & $1 \mathrm{a}$ & Thailand & \\
\hline A37 & I & $S$. Heidelberg & $1,4,[5], 12: r: 1,2$ & $\mathrm{He} 4$ & $9 \mathrm{i}$ & Colorado, USA & Turkey \\
\hline A38 & I & $S$. Heidelberg & $1,4,[5], 12: r: 1,2$ & He 5 & $9 \mathrm{i}$ & Arizona, USA & Turkey \\
\hline A39 & I & $S$. Heidelberg & $1,4,[5], 12: r: 1,2$ & $\mathrm{He} 7$ & $9 \mathrm{gi}$ & N. Carolina, USA & Human \\
\hline $\mathrm{A} 40$ & I & $S$. Heidelberg & $1,4,[5], 12: r: 1,2$ & $\mathrm{He} 8$ & $1 \mathrm{gi}$ & USA & \\
\hline $\mathrm{A} 41^{a *}$ & I & S. Paratyphi B & $1,4,[5], 12: b: 1,2$ & $\mathrm{~Pb} 1$ & $3 \mathrm{gh}$ & France & Human \\
\hline $\mathrm{A} 42^{a}$ & I & $S$. Paratyphi B & $1,4,[5], 12: b: 1,2$ & $\mathrm{~Pb} 1$ & $27 \mathrm{bg}$ & Scotland, UK & Human \\
\hline $\mathrm{A} 43^{a}$ & I & $S$. Paratyphi B & $1,4,[5], 12: b: 1,2$ & $\mathrm{~Pb} 1$ & $11 \mathrm{ghi}$ & Africa & Human \\
\hline $\mathrm{A} 44^{a}$ & I & $S$. Paratyphi B & $1,4,[5], 12: b: 1,2$ & $\mathrm{~Pb} 1$ & $19 \mathrm{gh}$ & Middle East & Human \\
\hline $\mathrm{A} 45^{\circ}$ & I & S. Paratyphi B & $1,4,[5], 12: b: 1,2$ & $\mathrm{~Pb} 1$ & & France & Cow \\
\hline $\mathrm{A} 46^{a}$ & I & S. Paratyphi B & $1,4,[5], 12: b: 1,2$ & $\mathrm{~Pb} 1 \mathrm{a}$ & $11 \mathrm{bgi}$ & Europe & Human \\
\hline $\mathrm{A} 47^{b}$ & I & S. Paratyphi B & $1,4,[5], 12: b: 1,2$ & $\mathrm{~Pb} 2$ & $9 \mathrm{a}^{2}$ & Scotland, UK & Sewage \\
\hline $\mathrm{A} 48^{b}$ & I & S. Paratyphi B & $1,4,[5], 12: b: 1,2$ & $\mathrm{~Pb} 2 \mathrm{a}$ & $1 \mathrm{dh}$ & Scotland, UK & Human \\
\hline $\mathrm{A} 49^{b}$ & I & $S$. Paratyphi B & $1,4,[5], 12: b: 1,2$ & $\mathrm{~Pb} 2 \mathrm{~b}$ & $1 \mathrm{~b}$ & UK & Sewage \\
\hline $\mathrm{A} 50^{b}$ & I & S. Paratyphi B & $1,4,[5], 12: b: 1,2$ & $\mathrm{~Pb} 3$ & $2 \mathrm{a}$ & Middle East & Food \\
\hline $\mathrm{A} 51^{b}$ & I & $S$. Paratyphi B & $1,4,[5], 12: b: 1,2$ & $\mathrm{~Pb} 3$ & $2 \mathrm{bg}$ & France & Human \\
\hline $\mathrm{A} 52^{\circ}$ & I & S. Paratyphi B & $1,4,[5], 12: b: 1,2$ & $\mathrm{~Pb} 3$ & & France & Cow \\
\hline $\mathrm{A} 53^{c}$ & I & S. Paratyphi B & $1,4,[5], 12: b: 1,2$ & $\mathrm{~Pb} 3$ & & France & Human \\
\hline $\mathrm{A} 54^{\circ}$ & I & S. Paratyphi B & $1,4,[5], 12: b: 1,2$ & $\mathrm{~Pb} 3$ & & France & Human \\
\hline $\mathrm{A} 55^{b}$ & I & $S$. Paratyphi B & $1,4,[5], 12: b: 1,2$ & $\mathrm{~Pb} 3 \mathrm{a}$ & $2 \mathrm{bg}$ & France & Human \\
\hline $\mathrm{A} 56^{b}$ & I & S. Paratyphi B & $1,4,[5], 12: b: 1,2$ & $\mathrm{~Pb} 4$ & $13 \mathrm{i}$ & France & Human \\
\hline $\mathrm{A} 57^{a}$ & I & S. Paratyphi B & $1,4,[5], 12: b: 1,2$ & $\mathrm{~Pb} 5$ & $11 \mathrm{i}$ & UK & Water \\
\hline $\mathrm{A} 58^{b}$ & I & S. Paratyphi B & $1,4,[5], 12: b: 1,2$ & $\mathrm{~Pb} 5 \mathrm{a}$ & $26 \mathrm{i}$ & France & Human \\
\hline $\mathrm{A} 59^{b}$ & I & $S$. Paratyphi B & $1,4,[5], 12: b: 1,2$ & $\mathrm{~Pb} 5 \mathrm{~b}$ & $10 \mathrm{i}$ & France & Human \\
\hline $\mathrm{A} 60^{b}$ & I & S. Paratyphi B & $1,4,[5], 12: b: 1,2$ & $\mathrm{~Pb} 5 \mathrm{c}$ & $9 \mathrm{bi}$ & France & Food \\
\hline $\mathrm{A} 61^{b}$ & I & S. Paratyphi B & $1,4,[5], 12: b: 1,2$ & $\mathrm{~Pb} 6$ & $1 \mathrm{a}$ & Scotland, UK & Water \\
\hline $\mathrm{A} 62^{b}$ & I & S. Paratyphi B & $1,4,[5], 12: b: 1,2$ & $\mathrm{~Pb} 7$ & $1 \mathrm{~g}$ & Africa & Human \\
\hline A63 & I & $S$. Muenchen & $6,8: \mathrm{d}: 1,2$ & Mu 1 & & France & Human \\
\hline A64 & I & $S$. Muenchen & $6,8: \mathrm{d}: 1,2$ & Mu 1 & & Kentucky, USA & Cow \\
\hline A65 & I & $S$. Muenchen & $6,8: \mathrm{d}: 1,2$ & Mu 1 & & Florida, USA & Chicken \\
\hline A66 & I & $S$. Muenchen & $6,8: \mathrm{d}: 1,2$ & $\mathrm{Mu} 1$ & & Massachusetts, USA & Human \\
\hline A67 & I & $S$. Muenchen & $6,8: \mathrm{d}: 1,2$ & Mu 1 & & Mexico & Human \\
\hline A68 & I & $S$. Muenchen & $6,8: \mathrm{d}: 1,2$ & Mu 1a & & France & Human \\
\hline
\end{tabular}


Table 1 (cont.)

\begin{tabular}{|c|c|c|c|c|c|c|c|}
\hline Strain & Subsp. & Serotype & Antigenic formula & ET & Biotype & Locality & Source \\
\hline A69 & I & $S$. Muenchen & $6,8: \mathrm{d}: 1,2$ & Mu 2 & & France & Human \\
\hline A70 & I & $S$. Muenchen & $6,8: \mathrm{d}: 1,2$ & Mu 3 & & France & Human \\
\hline A71 & I & $S$. Muenchen & $6,8: \mathrm{d}: 1,2$ & Mu 4 & & N. Carolina, USA & Human \\
\hline A72 & I & $S$. Muenchen & $6,8: \mathrm{d}: 1,2$ & $\mathrm{Mu} 4 \mathrm{a}$ & & France & Human \\
\hline \multicolumn{8}{|l|}{ SARB } \\
\hline B01 & I & S. Agona & 1,4,12:f,g,s:-- & Ag 1 & & Peru & \\
\hline B02 & I & S. Anatum & $3 \cdot 10: e, h: 1,6$ & An 1 & & Washington, USA & Human \\
\hline B03 & I & S. Brandenburg & 1,4,12:I,v:e,n,z15 & Ba 2 & $10 \mathrm{i}$ & Scotland, UK & \\
\hline B04 & I & S. Choleraesuis & $6,7:[c]: 1,5$ & Cs 1 & & Minnesota, USA & Swine \\
\hline B05 & I & S. Choleraesuis & $6,7:[\mathrm{c}]: 1,5$ & Cs 6 & & Switzerland & \\
\hline B06 & I & S. Choleraesuis & $6,7:[c]: 1,5$ & Cs 11 & & Thailand & \\
\hline B07 & I & S. Choleraesuis & $6,7:[\mathrm{c}]: 1,5 \mathrm{~s}$ & Cs 13 & & Australia & \\
\hline B08 & I & $S$. Decatur & $6,7: c: 1,5$ & Dt 1 & & France & \\
\hline B09 & I & $S$. Derby & $1,4,[5], 12: \mathrm{f}, \mathrm{g}:[1,2]$ & De 1 & & Oklahoma, USA & Avian \\
\hline B 10 & I & $S$. Derby & $1,4,[5], 12: \mathrm{f}, \mathrm{g}:[1,2]$ & De 13 & & Minnesota, USA & Swine \\
\hline B11 & I & $S$. Derby & $1,4,[5], 12: \mathrm{f}, \mathrm{g}:[1,2]$ & De 31 & & Pennsylvania, USA & Turkey \\
\hline B12 & I & $S$. Dublin & 1,9,12,[Vi]:g,p:-- & Du 1 & & Idaho, USA & Cattle \\
\hline B13 & I & S. Dublin & $1,9,12,[\mathrm{Vi}]: \mathrm{g}, \mathrm{p}:--$ & Du 3 & & France & Bovine \\
\hline B14 & I & $S$. Dublin & $1,9,12,[\mathrm{Vi}]: \mathrm{g}, \mathrm{p}:--$ & Du 2 & & Thailand & \\
\hline B15 & I & S. Duisburg & 1,4,12,27:d:e,n,z,15 & Di 1 & $9 \mathrm{i}$ & Scotland, UK & \\
\hline B16 & I & S. enteritidis & $1,9,12: \mathrm{g}, \mathrm{m}:[1,7]$ & En 1 & & Rhode Island, USA & \\
\hline B17 & I & S. enteritidis & $1,9,12: \mathrm{g}, \mathrm{m}:[1,7]$ & En 2 & & Brazil & \\
\hline B18 & I & S. enteritidis & $1,9,12: \mathrm{g}, \mathrm{m}:[1,7]$ & En 3 & & Connecticut, USA & \\
\hline B19 & I & S. enteritidis & $1,9,12: \mathrm{g}, \mathrm{m}:[1,7]$ & En 7 & & Switzerland & \\
\hline B20 & I & $S$. Emek & 8,20:g,m,s:-- & Em 1 & & Israel & \\
\hline B21 & I & S. Gallinarum & 1,9,12:--:-- & Ga 2 & & Connecticut, USA & Human \\
\hline B22 & I & $S$. Haifa & $1,4,[5], 12: z 10: 1,2$ & На 1 & $1 \mathrm{a}$ & Scotland, UK & \\
\hline B23 & I & $S$. Heidelberg & $1,4,[5], 12: r: 1,2$ & He 1 & $9 \mathrm{i}$ & Pennsylvania, USA & Chicken \\
\hline B24 & I & $S$. Heidelberg & $1,4,[5], 12: r: 1,2$ & $\mathrm{He} 3$ & $1 \mathrm{a}$ & Thailand & \\
\hline B25 & I & S. Indiana & $1,4,12: \mathrm{z}: 1,7$ & Id 1 & $1 \mathrm{a}$ & Scotland, UK & \\
\hline B26 & I & $S$. Infantis & $6,7: r: 1,5$ & In 1 & & N. Carolina, USA & Human \\
\hline B27 & I & $S$. Infantis & $6,7: r: 1,6$ & In 3 & & Senegal & \\
\hline B28 & I & S. Miami & $1,9,12: \mathrm{a}: 1,5$ & Mi 1 & & Georgia, USA & Human \\
\hline B29 & I & $S$. Miami & $1,9,12: \mathrm{a}: 1,6$ & Mi 5 & & French Guyana & Human \\
\hline B30 & I & S. Montevideo & $6,7: \mathrm{g}, \mathrm{m},[\mathrm{p}], \mathrm{s}:[1,2,7]$ & Mo 1 & & Georgia, USA & Human \\
\hline B31 & I & S. Montevideo & $6,7: \mathrm{g}, \mathrm{m},[\mathrm{p}], \mathrm{s}:[1,2,7]$ & Mo 6 & & Florida, USA & Human \\
\hline B32 & I & $S$. Muenchen & $6,8: \mathrm{d}: 1,2$ & Mu 1 & & Lab strain & \\
\hline B33 & I & $S$. Muenchen & $6,8: \mathrm{d}: 1,2$ & Mu 2 & & France & Human \\
\hline B34 & I & $S$. Muenchen & $6,8: \mathrm{d}: 1,2$ & Mu 3 & & France & Human \\
\hline B35 & I & $S$. Muenchen & $6,8: \mathrm{d}: 1,2$ & $\mathrm{Mu} 4$ & & N. Carolina, USA & Human \\
\hline B36 & I & $S$. Newport & $6,8: \mathrm{e}, \mathrm{h}: 1,2$ & $\mathrm{~Np} 8$ & & N. Carolina, USA & Human \\
\hline B37 & I & $S$. Newport & $6,8: \mathrm{e}, \mathrm{h}: 1,3$ & $\mathrm{~Np} 11$ & & Mexico & Human \\
\hline B38 & I & $S$. Newport & $6,8: \mathrm{e}, \mathrm{h}: 1,4$ & $\mathrm{~Np} 15$ & & Massachusetts, USA & Snake \\
\hline B39 & I & S. Panama & $1,9,12: \mathrm{I}, \mathrm{v}: 1,5$ & Pn 1 & & Italy & \\
\hline B40 & I & S. Panama & $1,9,12: \mathrm{I}, \mathrm{V}: 1,6$ & Pn 2 & & N. Carolina, USA & Human \\
\hline B41 & I & S. Panama & $1,9,12: \mathrm{I}, \mathrm{v}: 1,7$ & Pn 12 & & N. Carolina, USA & Human \\
\hline B42 & I & S. Paratyphi A & $1,2,12: \mathrm{a}:[1,5]$ & $\mathrm{Pa} 1$ & & Lab strain & \\
\hline $\mathrm{B} 43^{a}$ & I & S. Paratyphi B & $1,4,[5], 12: b:[1,2]$ & $\mathrm{Pb} 1$ & $3 \mathrm{gh}$ & France & Human \\
\hline $\mathrm{B} 44^{b}$ & I & S. Paratyphi B & $1,4,[5], 12: \mathrm{b}:[1,2]$ & $\mathrm{Pb} 3$ & $2 \mathrm{a}$ & Middle East & Food \\
\hline $\mathrm{B} 45^{\alpha}$ & I & $S$. Paratyphi B & $1,4,[5], 12: b:[1,2]$ & $\mathrm{Pb} 4$ & $3 \mathrm{I}$ & France & Human \\
\hline $\mathrm{B} 46^{a}$ & I & S. Paratyphi B & $1,4,[5], 12: \mathrm{b}:[1,2]$ & $\mathrm{Pb} 5$ & $11 \mathrm{i}$ & UK & Water \\
\hline $\mathrm{B} 47^{b}$ & I & $S$. Paratyphi B & $1,4,[5], 12: b:[1,2]$ & $\mathrm{Pb} 7$ & $1 \mathrm{~g}$ & Africa & Human \\
\hline B48 & I & S. Paratyphi C & $6,7,[\mathrm{Vi}]: \mathrm{c}: 1,5$ & Pc 1 & & France & \\
\hline B49 & I & S. Paratyphi C & $6,7,[\mathrm{Vi}]: \mathrm{c}: 1,6$ & Pc 2 & & France & Human \\
\hline B50 & I & S. Paratyphi C & $6,7,[\mathrm{Vi}]: \mathrm{c}: 1,7$ & $\operatorname{Pc} 4$ & & France & Human \\
\hline B51 & I & S. Pullorum & 1,9,12:--:-- & Pu 3 & & Germany & \\
\hline B52 & I & $S$. Pullorum & 1,9,12:--:-- & Pu 4 & & Germany & \\
\hline B53 & I & $S$. Reading & $1,4,[5], 12: e, h: 1,5$ & $\operatorname{Re} 1$ & $2 \mathrm{j}$ & Scotland, UK & \\
\hline B54 & I & $S$. Rubislaw & $11: r: e, n, x$ & Ru 1 & & Lab strain & \\
\hline B55 & I & S. Saintpaul & $1,4,[5], 12: e, h: 1,2$ & Sp 3 & & Texas, USA & Human \\
\hline B56 & I & S. Saintpaul & $1,4,[5], 12: \mathrm{e}, \mathrm{h}: 1,2$ & $\mathrm{Sp} 4$ & & Florida, USA & Human \\
\hline B57 & I & S. Schwarzengrund & $1,4,12,27: \mathrm{d}: 1,7$ & Sw 1 & $10 \mathrm{i}$ & Scotland, UK & \\
\hline B58 & I & $S$. Sendai & $1,9,12: \mathrm{a}: 1,5$ & Se 1 & & California, USA & Human \\
\hline B59 & I & $S$. Senftenberg & $1,3,19: \mathrm{g},[\mathrm{s}], \mathrm{t}:--$ & Sf 1 & & Maryland, USA & Chicken \\
\hline B 60 & I & $S$. Stanley & $1,4,[5], 12,27: \mathrm{d}: 1,2$ & St 1 & 26 bei & Scotland, UK & \\
\hline B61 & I & $S$. Stanleyville & $1,4,[5], 12,27: z 4, \mathrm{z} 12:[1,2]$ & Sv 2 & $9 \mathrm{i}$ & Scotland, UK & \\
\hline B62 & I & $S$. Thompson & $6,7: \mathrm{k}: 1,5$ & Th 1 & & Florida, USA & Human \\
\hline B63 & I & S. typhi & $9,12,[\mathrm{Vi}]: \mathrm{d}:[\mathrm{Z} 66]$ & Tp 1 & & Dakar, Senegal & \\
\hline B64 & I & S. typhi & $9,12,[\mathrm{Vi}]: \mathrm{d}:[\mathrm{Z} 66]$ & $\mathrm{Tp} 2$ & & Dakar, Senegal & \\
\hline B65 & I & S. typhimurium & $1,4,[5], 12: \mathrm{i}: 1,2$ & $\operatorname{Tm} 1$ & $1 \mathrm{f}$ & Mexico & Human \\
\hline B66 & I & S. typhimurium & $1,4,[5], 12: \mathrm{i}: 1,2$ & $\operatorname{Tm} 7$ & $1 \mathrm{a}$ & California, USA & Parrot \\
\hline B67 & I & S. typhimurium & $1,4,[5], 12: \mathrm{i}: 1,2$ & $\operatorname{Tm} 12$ & $29 \mathrm{~b}$ & France & \\
\hline B68 & I & S. typhimurium & $1,4,[5], 12: \mathrm{i}: 1,2$ & $\operatorname{Tm} 23$ & & Oregon, USA & Heron \\
\hline B69 & I & $S$. Typhisuis & $6,7: c: 1,5$ & Ts 1 & & California, USA & Swine \\
\hline
\end{tabular}


Table 1 (cont.)

\begin{tabular}{|c|c|c|c|c|c|c|c|}
\hline Strain & Subsp. & Serotype & Antigenic formula & ET & Biotype & Locality & Source \\
\hline B70 & I & $S$. Typhisuis & $6,7: c: 1,6$ & Ts 3 & & Iowa, USA & Swine \\
\hline B71 & I & $S$. Wien & $1,4,12,27: \mathrm{b}: 1: \mathrm{w}$ & Wi 1 & & France & Human \\
\hline B72 & I & $S$. Wien & $1,4,12,27: b: 1: w$ & Wi 2 & & France & Human \\
\hline \multicolumn{8}{|l|}{ SARC } \\
\hline $\mathrm{C} 01$ & I & S. typhimurium & $1,4,[5], 12: I: 1,2$ & & & England, UK & Human \\
\hline $\mathrm{C} 02$ & I & S. typhi & 9,12,[Vi]:d:[Z66] & Tp 1 & & Dakar, Senegal & \\
\hline $\mathrm{C} 03$ & II & $S$. Unnamed & $58: \mathrm{d}: \mathrm{z}_{6}$ & & & Massachusetts, USA & Human \\
\hline $\mathrm{C} 04$ & II & $S$. Unnamed & $42: f: g, t:-$ & & & & \\
\hline $\mathrm{C} 05$ & IIIa & & $62: z_{4}: z_{23}:-$ & & & Oregon, USA & Cornsnake \\
\hline $\mathrm{C} 06$ & IIIa & & $62: z: 36$ & & & California, USA & Human \\
\hline $\mathrm{C} 07$ & IIIb & & $50_{1,2,3}: \mathrm{k}: \mathrm{z}$ & & & Oregon, USA & Human \\
\hline $\mathrm{C} 08$ & IIIb & & $35[\mathrm{k}]: \mathrm{z}_{35}:-$ & & & California, USA & Human \\
\hline $\mathrm{C} 09$ & IV & & $45 \mathrm{a}, \mathrm{b}: \mathrm{g}: \mathrm{z}_{51}:-$ & & & Panama Canal Zone & Animal \\
\hline $\mathrm{C} 10$ & IV & $S$. Chameleon & $16: z_{4}: z_{32}:-$ & & & Illinois, USA & Human \\
\hline $\mathrm{C} 11$ & $\mathrm{~V}$ & S. Brookfield & $66: z_{41}:-$ & & & & Frog \\
\hline $\mathrm{C} 12$ & $\mathrm{~V}$ & $S$. Balboa & $48: z_{41}:-$ & & & USA & Parakeet \\
\hline $\mathrm{C} 13$ & VI & $S$. Vrindaban & $45: a: e, n, x$ & & & India & \\
\hline $\mathrm{C} 14$ & VI & $S$. Srinagar & $11: b: e, n, x$ & & & & \\
\hline $\mathrm{C} 15$ & VII & $S$. Seminole & $1,40: \mathrm{g}, \mathrm{z}_{51}$ & & & Tonga & \\
\hline $\mathrm{C} 16$ & VII & $S$. Unnamed & $40: z_{4}, z_{24}:-$ & & & Florida, USA & Human \\
\hline
\end{tabular}

* $S$. Paratyphi B biotypes are indicated as: $a$, Paratyphi B; $b$, Java; $c$, unknown.

Sigma-Genosys) and 0.65 U Taq DNA polymerase (Life Technologies). Touchdown PCR was performed as described by Desai et al. (1998) with the exception that the final extension step at $60{ }^{\circ} \mathrm{C}$ for $30 \mathrm{~min}$ was omitted to limit the addition of an $n+1$ adenine to FAFLP fragments by Taq DNA polymerase.

Fragment analysis. Gel separation and fragment analyses were performed as described previously (Grady et al., 1999). Fragments were sized with GeneScan version 3.1 software (Applied Biosystems) and these data were transferred to GenoTyper 2.5 (Applied Biosystems).

Electrophoregrams were screened visually for the presence or absence of polymorphic fragments, which were scored in a binary matrix and recorded as a text (tab-delimited) file in Excel 98 (Microsoft). Dice coefficients of similarity (Dice, 1945) were calculated using in-house software. Cluster analysis was performed using the neighbour-joining method (Saitou \& Nei, 1987) in the NEIGHBOR program of PHYLIP (Felsenstein, 1993) and then displayed in TREEVIEW version 1.5 (Page, 1996). A bootstrap tree (i.e. the generation of multiple datasets that are resampled versions of the input dataset) was produced in PAUP* (Swofford, 2000) using Nei-Li distances (Nei \& Li, 1979) and neighbour-joining cluster analysis (Saitou \& Nei, 1987) (data not shown). Where clusters and groupings of strains similar by FAFLP were observed, a 'cluster' was defined as those strains that both clustered by neighbour-joining and had high bootstrap support (i.e. $>75 \%$ ) in Fig. 4; a 'group' was defined as those strains that clustered by neighbour-joining but had less than $75 \%$ bootstrap support in Fig. 4 .

In silico FAFLP. In silico FAFLP analysis was performed as described by Arnold et al. (1999b) on the inv/spa genes of strains C09, C10, C11 and C12, respectively GenBank accession numbers U29357, U29358, U29355 and U29356 (Li et al., 1995; Boyd et al., 1996).

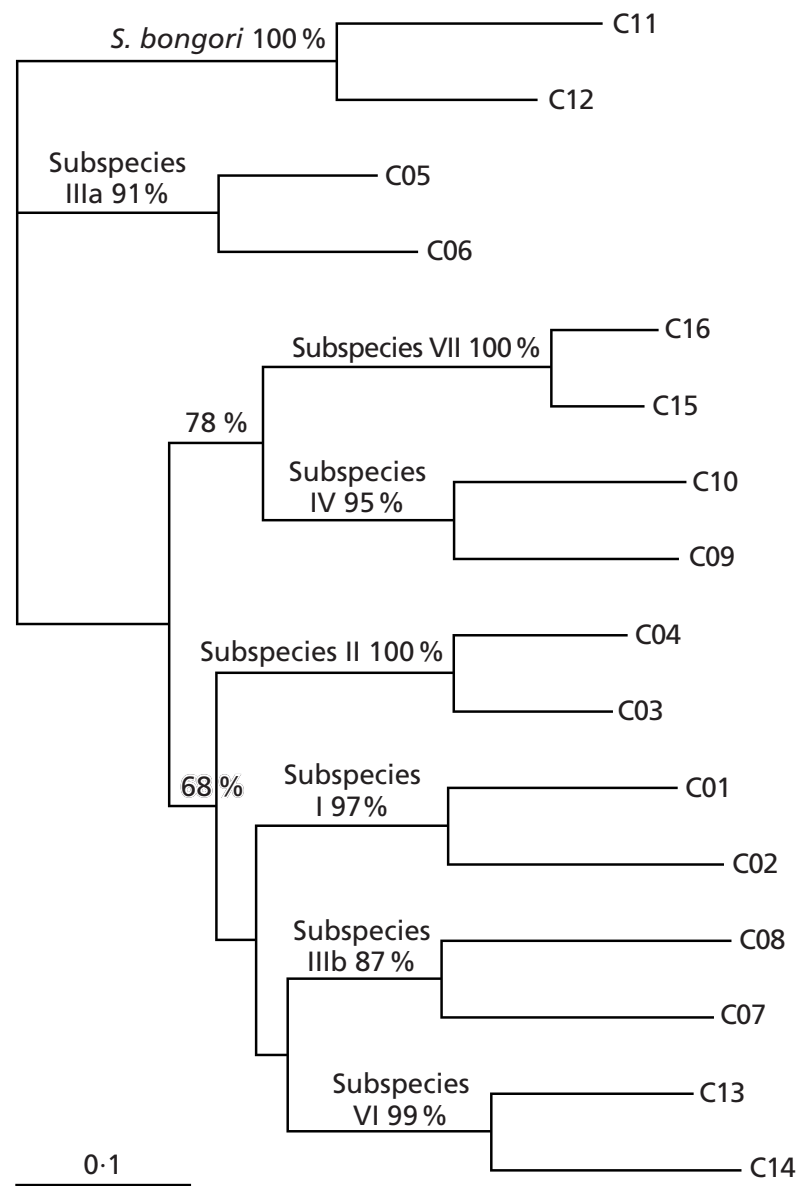

Fig. 1. Neighbour-joining tree of SARC strains. Bootstrap values were calculated in PAUP* using Nei-Li distances and neighbourjoining cluster analysis. The tree is rooted at $S$. bongori strains C11 and C12. Bar, $10 \%$ divergence. 


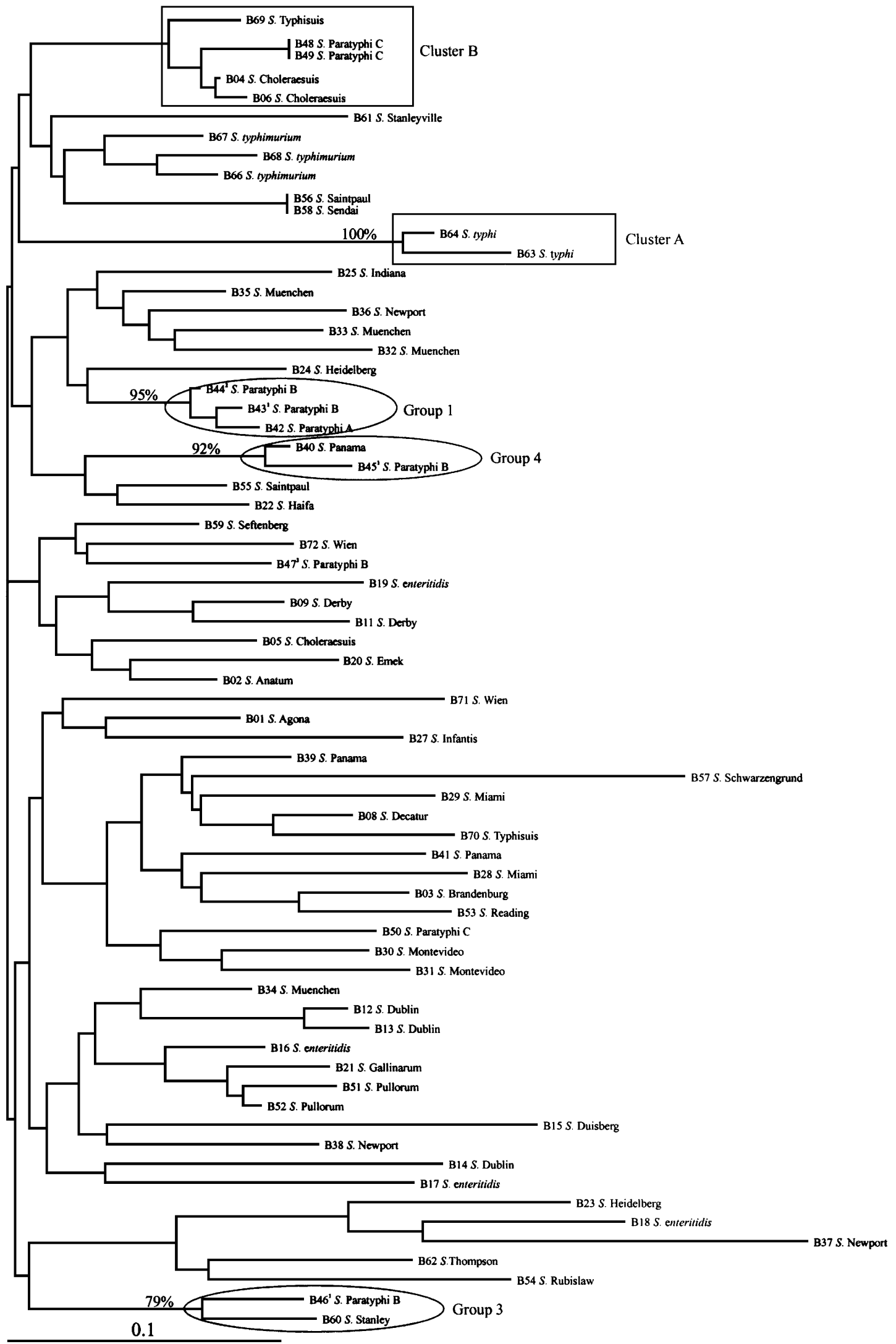

Fig. 2. Neighbour-joining tree of SARB strains. Bootstrap values were calculated as described for Fig. 1. See text for definition of clusters and groups. Bar, $10 \%$ divergence. A version of this tree showing various serotypes identified in colour is available as supplementary material in IJSEM Online (http://ijs.sgmjournals.org/). 


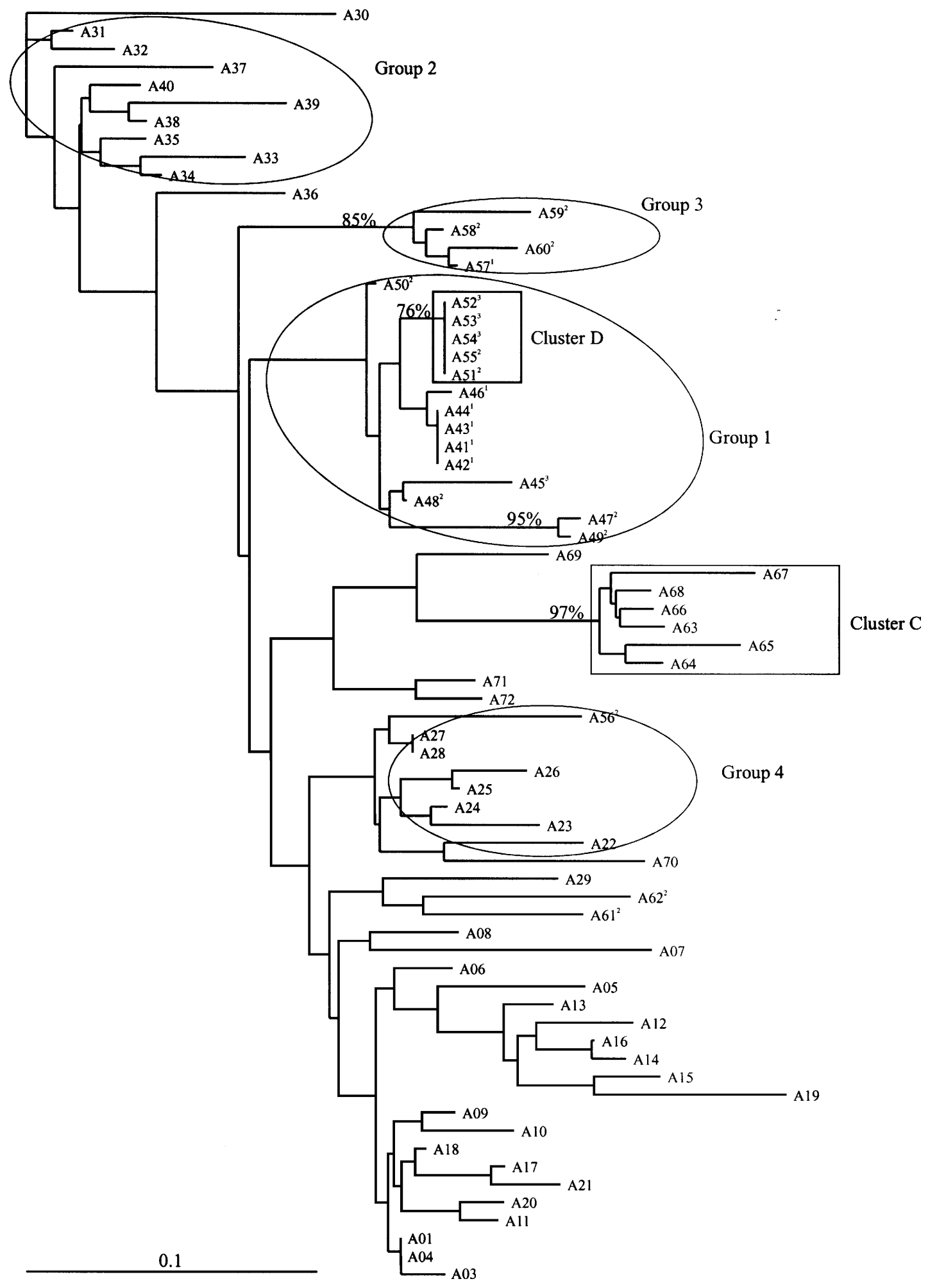

Fig. 3. Neighbour-joining tree of SARA strains. The tree is rooted at A30. Bootstrap values were calculated as described for Fig. 1. Clusters and groupings shown are defined in the text. Bar, $10 \%$ divergence. A version of this tree showing various serotypes identified in colour is available as supplementary material in IJSEM Online (http://ijs.sgmjournals.org/).

\section{RESULTS}

We analysed 183 polymorphic fragments, in the size range 101-350 bp, of the 155 SARA, B and C strains, and we found that no amplified fragment was common to all strains. There were 14 strain duplications between the collections and duplicates showed on average $99 \cdot 2 \%$ similarity (range $98-100 \%$ ). Figs $1-3$ respect- 


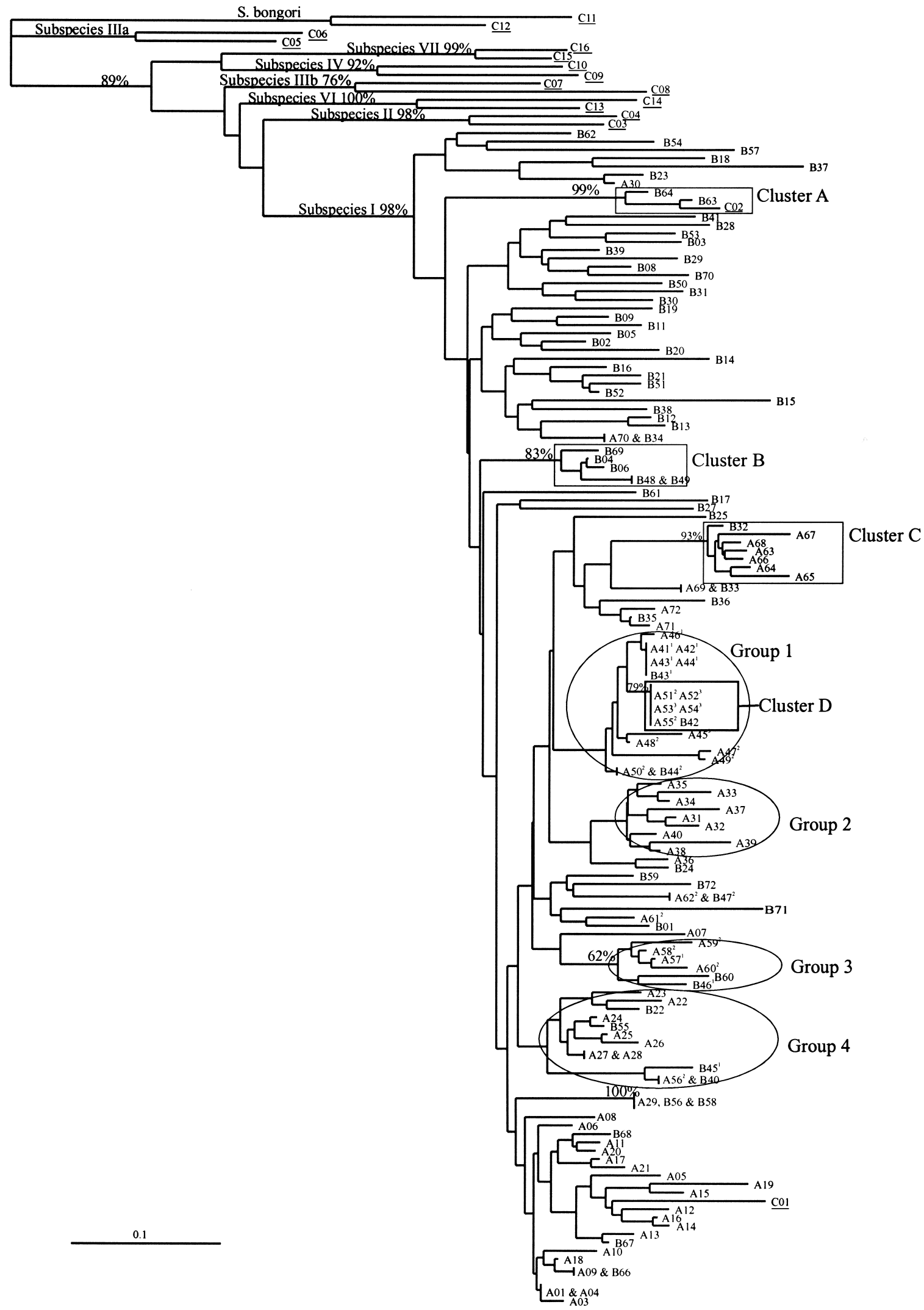

Fig. 4. Neighbour-joining tree of SARA, SARB and SARC strains. The tree is rooted at S. bongori strains C11 and C12. Bootstrap values were calculated as described for Fig. 1. SARC strains are underlined. Clusters and groupings shown are defined in the text. Bar, $10 \%$ divergence. A version of this tree showing various serotypes identified in colour is available as supplementary material in IJSEM Online (http://ijs.sgmjournals.org/). 
ively show FAFLP trees of SARC, SARB and SARA and Fig. 4 shows an FAFLP tree of all three reference collections combined.

\section{FAFLP analysis of the Salmonella subspecies (SARC)}

The strains from SARC fell into eight distinct pairs, consisting of two strains representing each of the seven MLEE defined subspecies and S. bongori (Fig. 1). There was high bootstrap support for these pairs, i.e. $99 \%$ for $\mathrm{C} 13$ and $\mathrm{C} 14$ (subspecies VI), $100 \%$ for $\mathrm{C} 15$ and $\mathrm{C} 16$ (subspecies VII), $100 \%$ for $\mathrm{C} 03$ and $\mathrm{C} 04$ (subspecies II), 95\% for C09 and C10 (subspecies IV), $97 \%$ for C01, C02 (subspecies I) and $87 \%$ for $\mathrm{C} 07$ and C08 (subspecies IIIb). There was some bootstrap support $(68 \%)$ for the separation of the subspecies that exhibit predominantly or exclusively diphasic expression of the flagella $\mathrm{Ag}$ (subspecies I, C01 and C02; II, C03 and C04; IIIb, C07 and C08; and VI, C13 and C14) from the monophasic subspecies (IIIa, C05 and $\mathrm{C} 06$; IV, $\mathrm{C} 09$ and $\mathrm{C} 10 ; S$. bongori $\mathrm{C} 11$ and $\mathrm{C} 12$; and VII, C15 and C16).

The two $S$. bongori strains, $\mathrm{C} 11$ and $\mathrm{C} 12$, were the most divergent by FAFLP, followed by the two strains of subspecies IIIa (C05 and C06). The strains of subspecies VII were divergent from those of subspecies IV, with 38 amplified-fragment differences between C10 (subspecies IV) and C15 (subspecies VII). Only 18 amplified fragments were common to all four strains. However, the two subspecies clustered to each other more than with other subspecies, with $78 \%$ bootstrap support.

\section{FAFLP analysis of Salmonella subspecies I (SARB)}

Both the strains of S. typhi were found in Cluster A, with high bootstrap support (100\%) (Fig. 2). Cluster B consisted of two strains of Salmonella Choleraesuis (B04 and B06) and one of Salmonella Typhisuis (B69), all three of which were epidemiologically unrelated strains from swine, as well as two strains of Salmonella Paratyphi C (B48 and B49). The latter two strains had identical FAFLP profiles. There were seven amplifiedfragment differences between the cluster B strains and this cluster had $88 \%$ bootstrap support. The third strains of $S$. Choleraesuis (B05) and of $S$. Paratyphi C (B50) were very divergent from the two strains found in cluster B, as was the second strain of $S$. Typhisuis (B70).

\section{FAFLP analysis of the S. typhimurium complex (SARA)}

Six of the ten strains of Salmonella Muenchen (A63A68) clustered together (cluster C) and were divergent from the others of this serotype (Fig. 3). This cluster had $97 \%$ bootstrap support and there were 12 amplified fragments different between the six strains. Three of the remaining four strains of $S$. Muenchen also grouped with cluster $\mathrm{C}$, but without bootstrap support. The strains of $S$. Paratyphi B were found to be diverse by FAFLP, although tight clustering was observed for some groups of these strains. Fifteen of the $22 S$. Paratyphi B strains in SARA were found in Group 1, four in Group 3 and one in Group 4. The remaining two strains of this serotype were very diverse by FAFLP from the clusters and groups described above. Cluster D in group 1 contained five $S$. Paratyphi B strains (A51-A55) that were identical by FAFLP, and cluster D had $76 \%$ bootstrap support. There were a further four $S$. Paratyphi B strains (A41-A44) that were identical by FAFLP in group 1; however, these strains had no bootstrap support and were therefore not considered a cluster. The four strains of $S$. Paratyphi B (A57-A60) in Group 3 had 85\% bootstrap support. There were six amplified fragments different between the four strains in group 3 .

Group 2 was composed of nine strains of Salmonella Heidelberg (A30-A35, A37, A39 and A40); however, there was no bootstrap support for the clustering of these isolates. The remaining strain of $S$. Heidelberg (A36) was found near group 2. Six of the seven strains of serotype Salmonella Saintpaul in SARA formed Group 4 (A22-A28), together with one strain of $S$. Muenchen (A70), though there was no bootstrap support for clustering of these strains. The remaining strain of $S$. Saintpaul (A29) clustered away from this group. The strains of $S$. typhimurium (A01-21) were found near each other; however, there was no bootstrap support for clustering or grouping of these strains.

\section{SARA, B and C combined}

The dendrogram of SARA, B and C strains is rooted at $\mathrm{C} 11$ and $\mathrm{C} 12$ (S. bongori) (Fig. 4). Cluster A contained all three $S$. typhi strains, with only seven amplified fragments different between them. This cluster, supported by a bootstrap value of $99 \%$, also appeared very divergent from other serotypes by FAFLP. Cluster B, also seen in Fig. 2, remained in Fig. 4 when data from all three collections were combined, but with slightly lower bootstrap support than described previously $(83 \%)$. Cluster C consisted of $S$. Muenchen strains and is also seen in Fig. 3. Found near cluster C, but not supported by bootstrap analysis, were a further five strains of $S$. Muenchen (A69, A71, B33, B35 and B36). The only remaining strain of this serotype (B34) was very divergent from this cluster.

Seventeen $S$. Paratyphi B strains and one $S$. Paratyphi A strain formed group 1 (also seen in Figs 2 and 3). Within this group was cluster D (also seen in Figs 2 and 3).

Group 2 consisted of nine of the $13 S$. Heidelberg strains. One strain of $S$. Heidelberg (represented by A36 and B24) was found near this group, while the two remaining strains (A30 and B23) now clustered with $S$. enteritidis B18 and Salmonella Newport B37, with 
$80 \%$ bootstrap support. Five strains of $S$. Paratyphi B (A57-A60 and B46) and one of Salmonella Stanley (B60) constituted Group 3. This group had $62 \%$ bootstrap support and there were 13 amplified fragments different between these strains.

Twelve strains of serotypes $S$. Saintpaul, Salmonella Haifa, S. Paratyphi B and Salmonella Panama formed Group 4, but there was no bootstrap support for clustering of these strains. Two strains of $S$. Saintpaul (A29 and B56) and Salmonella Sendai B58 clustered separately from this group, with $100 \%$ bootstrap support. These strains had identical Eco + $0 \mathrm{Mse}+\mathrm{TA}$ FAFLP profiles, but B58 had an extra amplified $E c o+0 M s e+T$ fragment at 200 bp (data not shown), not present in A29 or B56. Strains of $S$. Paratyphi B were found to be very diverse by FAFLP. As well as the 17 strains found in Group 1, there were five in Group 3 and two in Group 4. The remaining two strains of this serotype were very diverse by FAFLP from the groups and clusters described above. $S$. enteritidis strains (B16-B19) were also very diverse by FAFLP, with strains being found throughout the tree.

\section{In silico FAFLP}

In silico FAFLP analysis demonstrated that no Eco RI or $M s e+$ TA restriction sites were present in the inv/spa genes of C09 (subspecies IV), C15 and C16 (both subspecies VII). One Mse + TA site was present in C10 (subspecies IV) at $-180 \mathrm{bp}$ from the $3^{\prime}$ end. However, there were no Eco + 0 Mse + TA amplified fragments above this size present only in this strain, and not in the others of subspecies IV or VII. It is therefore unlikely that there is an EcoRI restriction site in close proximity to give an amplified fragment that would be detected in this analysis.

\section{DISCUSSION}

The results shown here correspond more closely with the genetic structure of Salmonella using the medical microbiology system of taxonomic definition. This consists of two Salmonella species, S. bongori and $S$. choleraesuis (or $S$. enterica), the latter of which is further subdivided into six subspecies. Classical Salmonella taxonomy recognizes these two species, but also includes $S$. enteritidis, $S$. typhimurium and $S$. typhi as Salmonella species. FAFLP shows that $S$. enteritidis and $S$. typhimurium are found within the main cluster of subspecies I strains and do not appear to be sufficiently divergent to be regarded as separate $\mathrm{Sal}$ monella species. S. typhi was more diverse by FAFLP, but still not necessarily sufficiently divergent to be considered a separate species. These FAFLP results are supported by MLEE, which also includes these serotypes in subspecies I (Boyd et al., 1993).

MLEE and sequencing studies have been used to establish the population genetic frameworks (i.e. macro-variation) for Salmonella. Many MLEE studies have been performed, but there is no consensus among researchers regarding which enzymes should be chosen, with the result that over 40 housekeeping enzymes have been used in various studies (Selander et al., 1986, 1990a, b, 1992; Beltran et al., 1988, 1991; Reeves et al., 1989; Boyd et al., 1993, 1996; Osaka et al., 1999). Sequencing studies have been based on only a fraction of the genes encoding these proteins (Nelson \& Selander, 1992, 1994; Boyd et al., 1994, 1996; Nelson et al., 1997; Wang et al., 1997). Accurate comparison of the FAFLP results with those obtained using MLEE is therefore difficult, especially as the three reference collections have never been brought together before. However, we find that our FAFLP results, while supporting a number of findings from MLEE studies, are more closely related to the earlier hybridization studies and more recent sequencing studies of housekeeping genes and invasion genes (Boyd et al., 1996).

\section{SARC}

FAFLP has been successful in distinguishing the MLEE-defined subspecies of Salmonella, each pair being found clustered together. These FAFLP results also support MLEE and DNA hybridization results in confirming that $S$. bongori is the most divergent form of Salmonella and should be regarded as a separate species (Reeves et al., 1989; Boyd et al., 1996). In addition, FAFLP analysis shows that the two strains of subspecies IIIa are the second most divergent form of Salmonella. This finding corresponds to sequencing studies of housekeeping and invasion genes, where this subspecies is second only to $S$. bongori in its divergence from the other subspecies. This is not, however, reflected in the MLEE findings, where subspecies IIIa is not divergent and is found clustering with subspecies I, IIIb and VI (Boyd et al., 1996).

FAFLP supports the MLEE data in separating the strains of subspecies VII from those of subspecies IV, which are grouped together by biotype. Sequence analysis of five housekeeping genes revealed that the degree of nucleotide divergence between subspecies IV and VII was at least as great as that between subspecies I and VI or II and IIIb. However, little difference was observed in the sequence of their invasion genes (Boyd et al., 1996). On the basis of these findings, Boyd et al. (1996) proposed a model of evolution based on a mosaic genome structure for strains of subspecies VII. They proposed that the genome of the group VII strains is a mosaic of large chromosomal segments with different evolutionary histories, the major part of the chromosome originating from an old lineage that has achieved a level of genetic differentiation similar to the other subspecies of $S$. choleraesuis. Presumably, it is this segment of the genome that is being assayed by MLEE and includes the five housekeeping genes. In this study, we have identified 18 polymorphic amplified fragments common to all four strains of these two subspecies. Recent horizontal transfer of gene segments including the inv/spa region from subspecies IV 
to VII could account for the similarity in this region between these two groups. In silico FAFLP analysis of the inv/spa genes of the two strains of subspecies IV and VII showed that no amplified fragments would be detected from these loci using the primer combination $E c o+0$ and $M s e+T A$, indicating that FAFLP distinguishes between groups IV and VII in a manner similar to that by MLEE and the study involving the housekeeping genes.

The mono- and diphasic subspecies of Salmonella clustered separately. This pattern was also observed in the clustering of Salmonella based on the nucleotide sequences of housekeeping and invasion genes (Boyd et al., 1996). Selander \& Nelson (1996) proposed an evolutionary model to explain these findings: approximately 160-180 million years ago, E. coli and $S$. choleraesuis evolved from a common ancestor. E. coli developed as a commensal and opportunistic pathogen of mammals and birds, while Salmonella was associated with reptiles. Subsequently, members of Salmonella evolved as intracellular pathogens through the acquisition of invasion and other genes that mediate invasion of the host epithelial cells. By providing an increased ability to evade the host immune system, the development of diphasic variation in a lineage ancestral to subspecies I, II, IIIb and VI may have been essential to allow the exploitation of a new ecological niche in mammals and birds as a pathogen. Subspecies I then became highly specialized for mammals and birds, with some serotypes adapting to a single host species, e.g. S. typhi. However, some serotypes of subspecies I and II have since reverted to monophasic expression of the flagellar genes.

\section{SARA and SARB (Salmonella subspecies I)}

Of the polyphyletic serotypes described by Boyd et al. (1993), three (S. enteritidis, $S$. Newport and $S$. Typhisuis) are highly divergent by FAFLP when all three reference collections are brought together. Of the remaining serotypes, five ( $S$. Choleraesuis, $S$. Muenchen, $S$. Paratyphi B, $S$. Paratyphi C and $S$. Saintpaul) have some ETs that are found in groups or clusters, while other serotypes have separate, divergent ETs. For example, the two ETs of Salmonella Derby (B09 and B10) clustered together by FAFLP with high bootstrap support, but these were identified as being highly divergent by MLEE, belonging to two very distantly related lineages, I and III (Beltran et al., 1988).

$S$. Paratyphi B ETs were found in FAFLP groups 1, 3 and 4 , but the remaining ETs of this serotype were highly divergent and fall outside these groupings. $S$. Paratyphi B strains can be separated according to biotype. Strains that are D-tartrate-positive and slime wall-negative are designated $S$. Paratyphi biotype Java, whereas those that are D-tartrate-negative and slime wall-positive are designated $S$. Paratyphi B biotype Paratyphi B (Duguid et al., 1975; Barker et al., 1988). Strains designated as $S$. Paratyphi B biotype
Java are highly divergent by FAFLP, with two strains being found in cluster D, four in Group 3, two in Group 4 and a further three not falling into any of the groups or clusters. S. Paratyphi B biotype Paratyphi B, on the other hand, appears to be a more homogeneous group of strains, the majority of which are identical by FAFLP and are found in Group 1. A further strain of biotype Paratyphi B (ET Pb 5) is found in Group 3. These results correspond well to those of MLEE. Most of the biotype Paratyphi B strains, which cause enteric fever, are members of a globally distributed clone (ET $\mathrm{Pb}$ 1). In contrast, the biotype Java strains, which generally cause only gastroenteritic disease, are highly heterogeneous (Selander et al., 1990a). This phenomenon is thought to be influenced by host adaptation, with the MLEE results suggesting that clones of human-adapted serotypes, e.g. S. typhi and S. Paratyphi $\mathrm{C}$, are fewer in number and have less diverse genotypes than those that have a broad host range, e.g. $S$. Heidelberg, S. typhimurium and S. enteritidis (Selander et al., 1990a). FAFLP shows that the three strains of $S$. typhi cluster together, as do two strains of $S$. Paratyphi C, while the third is found to be more divergent. In contrast, strains of $S$. Heidelberg, $S$. typhimurium and $S$. enteritidis, in particular, are highly divergent by FAFLP.

\section{FAFLP versus MLEE}

Changes in the amino acid sequence that do not affect the net electrostatic charge of the protein will not be detected by MLEE. Variation in the nucleotide sequence that does not alter the amino acid sequence will also go undetected by MLEE. It is estimated that $10-20 \%$ of sequence variation could therefore go undetected (Selander et al., 1986). In contrast, the migration rate of an FAFLP fragment is determined by its size, which is in turn determined by the position of specific restriction sites and the surrounding sequences. Mutations such as insertions or deletions will be detected by FAFLP. However, substitutions might go undetected unless they occur in the restriction site or in the selective bases surrounding it, or if they have an effect on the secondary structure of the fragment that alters the rate of migration in some way. Therefore, a major advantage of FAFLP above MLEE and current sequence-based approaches is that the genome is sampled throughout its length at both variable and non-variable regions, with little or no bias. By contrast, methods such as MLEE, MLST and other sequencebased approaches only sample small, highly conserved and slowly evolving segments of the genome.

In silico FAFLP modelling on the sequenced E. coli K12 MG 1655 genome revealed that FAFLP, using the selective primer combination Eco + $0 \mathrm{Mse}+\mathrm{TA}$, sampled $0.25 \%$ of the genome (Arnold et al., 1999b). We have determined for this study, using the same selective primer combination, that we have the potential to sample approximately $0.85 \%$ of a given genome (based on the $S$. typhimurium genome size of $4.5 \mathrm{Mb}$ ). How- 
ever, each individual strain contains only a proportion of the fragments included in this study. For $S$. typhimurium $\mathrm{C} 01$, therefore, $0 \cdot 19 \%$ of the genome is being sampled.

We find that FAFLP is successful in discriminating between the subspecies of Salmonella and that it compares well with MLEE in identifying clonal lineages. We propose that FAFLP is a powerful tool for population studies, as it provides an unbiased view of whole-genome variation in natural populations of Salmonella.

\section{ACKNOWLEDGEMENTS}

The authors would like to thank Drs Philip Mortimer and Julie Logan for critical reading of this manuscript.

\section{REFERENCES}

Arnold, C., Metherell, L., Clewley, J.P. \& Stanley, J. (1999a). Predictive modelling of fluorescent AFLP: a new approach to the molecular epidemiology of E. coli. Res Microbiol 150, 33-44.

Arnold, C., Metherell, L., Willshaw, G., Maggs, A. \& Stanley, J. (1999b). Predictive fluorescent amplified-fragment length polymorphism analysis of Escherichia coli: high-resolution typing method with phylogenetic significance. J Clin Microbiol 37, 1274-1279.

Barker, R. M., Kearney, G. M., Nicholson, P., Blair, A. L., Porter, R. C. \& Crichton, P. B. (1988). Types of Salmonella paratyphi B and their phylogenetic significance. J Med Microbiol 26, 285-293.

Beltran, P., Musser, J. M., Helmuth, R. \& 8 other authors (1988). Toward a population genetic analysis of Salmonella: genetic diversity and relationships among strains of serotypes $S$. choleraesuis, $S$. derby, $S$. dublin, S. enteritidis, $S$. heidelberg, S. infantis, $S$. newport, and $S$. typhimurium. Proc Natl Acad Sci U S A 85, 7753-7757.

Beltran, P., Plock, S. A., Smith, N. H., Whittam, T. S., Old, D. C. \& Selander, R. K. (1991). Reference collection of strains of the Salmonella typhimurium complex from natural populations. J Gen Microbiol 137, 601-606.

Boyd, E. F., Wang, F.-S., Beltran, P., Plock, S. A., Nelson, K. \& Selander, R. K. (1993). Salmonella reference collection B (SARB): strains of 37 serovars of subspecies I. J Gen Microbiol 139, 1125-1132.

Boyd, E. F., Nelson, K., Wang, F.-S., Whittam, T. S. \& Selander, R. K. (1994). Molecular genetic basis of allelic polymorphism in malate dehydrogenase $(\mathrm{mdh})$ in natural populations of Escherichia coli and Salmonella enterica. Proc Natl Acad Sci U S A 91, 1280-1284.

Boyd, E. F., Wang, F.-S., Whittam, T. S. \& Selander, R. K. (1996). Molecular genetic relationships of the salmonellae. Appl Environ Microbiol 62, 804-808

Briggs, C. E. \& Fratamico, P. M. (1999). Molecular characterization of an antibiotic resistance gene cluster of Salmonella typhimurium DT104. Antimicrob Agents Chemother 43, 846-849.

Desai, M., Tanna, A., Wall, R., Efstratiou, A., George, R. \& Stanley, J. (1998). Fluorescent amplified-fragment length polymorphism analysis of an outbreak of group A streptococcal invasive disease. $J$ Clin Microbiol 36, 3133-3137.

Dice, L. R. (1945). Measures of the amount of ecological association between species. Ecology 26, 297-302.

Duguid, J. P., Anderson, E. S., Alfredsson, G. A., Barker, R. \& Old, D. C. (1975). A new biotyping scheme for Salmonella typhimurium and its phylogenetic significance. J Med Microbiol 8, 149-166.

Enright, M. C. \& Spratt, B. G. (1998). A multilocus sequence typing scheme for Streptococcus pneumoniae: identification of clones associated with serious invasive disease. Microbiology 144, 3049-3060.

Enright, M. C., Day, N. P., Davies, C. E., Peacock, S. J. \& Spratt, B. G. (2000). Multilocus sequence typing for characterization of methicillin-resistant and methicillin-susceptible clones of Staphylococcus aureus. J Clin Microbiol 38, 1008-1015.

Ezquerra, E., Burnens, A., Jones, C. \& Stanley, J. (1993). Genotypic typing and phylogenetic analysis of Salmonella paratyphi $B$ and $S$. java with IS200. J Gen Microbiol 139, 2409-2414.

Felsenstein, J. (1993). PHYLIP (Phylogeny Inference Package). Distributed by the author. Department of Genetics, University of Washington, Seattle, USA.

Grady, R., Desai, M., O’Neill, G., Cookson, B. \& Stanley, J. (1999). Genotyping of epidemic methicillin-resistant Staphylococcus aureus phage type 15 isolates by fluorescent amplified-fragment length polymorphism analysis. J Clin Microbiol 37, 3198-3203.

Kariuki, S., Cheesbrough, J., Mavridis, A. K. \& Hart, C. A. (1999a). Typing of Salmonella enterica serotype Paratyphi C isolates from various countries by plasmid profiles and pulsed-field gel electrophoresis. J Clin Microbiol 37, 2058-2060.

Kariuki, S., Gilks, C., Kimari, J., Muyodi, J., Waiyaki, P. \& Hart, C. A. (1999b). Analysis of Salmonella enterica serotype Typhimurium by phage typing, antimicrobial susceptibility and pulsed-field gel electrophoresis. J Med Microbiol 48, 1037-1042.

Katiyar, S. K., Chandel, G., Tan, Y. \& 8 other authors (2000). Biodiversity of Asian rice gall midge (Orseolia oryzae Wood Mason) from five countries examined by AFLP analysis. Genome 43, 322-332.

Keiper, F. J. \& McConchie, R. (2000). An analysis of genetic variation in natural populations of Sticherus flabellatus [R. Br. (St John)] using amplified fragment length polymorphism (AFLP) markers. Mol Ecol $\mathbf{9}$, 571-581.

Laconcha, I., Baggesen, D. L., Rementeria, A. \& Garaizar, J. (2000). Genotypic characterisation by PFGE of Salmonella enterica serotype Enteritidis phage types 1, 4, 6, and 8 isolated from animal and human sources in three European countries. Vet Microbiol 75, 155-165.

Li, J., Ochman, H., Groisman, E. A., Boyd, E. F., Solomon, F., Nelson, K. \& Selander, R. K. (1995). Relationship between evolutionary rate and cellular location among the Inv/Spa invasion proteins of Salmonella enterica. Proc Natl Acad Sci U S A 92, 7252-7256.

Maiden, M. C., Bygraves, J. A., Feil, E. \& 10 other authors (1998). Multilocus sequence typing: a portable approach to the identification of clones within populations of pathogenic microorganisms. Proc Natl Acad Sci U S A 95, 3140-3145.

Millemann, Y., Gaubert, S., Remy, D. \& Colmin, C. (2000). Evaluation of IS200-PCR and comparison with other molecular markers to trace Salmonella enterica subsp. enterica serotype typhimurium bovine isolates from farm to meat. J Clin Microbiol 38, 2204-2209.

Nei, M. \& Li, W. H. (1979). Mathematical model for studying genetic variation in terms of restriction endonucleases. Proc Natl Acad Sci US A 76, 5269-5273.

Nelson, K. \& Selander, R. K. (1992). Evolutionary genetics of the proline permease gene (put $\mathrm{P})$ and the control region of the proline utilization operon in populations of Salmonella and Escherichia coli. $J$ Bacteriol 174, 6886-6895.

Nelson, K. \& Selander, R. K. (1994). Intergeneric transfer and recombination of the 6-phosphogluconate dehydrogenase gene ( $g n d)$ in enteric bacteria. Proc Natl Acad Sci U S A 91, 10227-10231.

Nelson, K., Wang, F. S., Boyd, E. F. \& Selander, R. K. (1997). Size and sequence polymorphism in the isocitrate dehydrogenase kinase/ phosphatase gene (aceK) and flanking regions in Salmonella enterica and Escherichia coli. Genetics 147, 1509-1520.

Ochman, H. \& Selander, R. K. (1984). Standard reference strains of Escherichia coli from natural populations. J Bacteriol 157, 690-693.

Old, D. C., Rankin, S. C. \& Crichton, P. B. (1999). Assessment of strain relatedness among Salmonella serotypes Salinatis, Duisburg, and Sandiego by biotyping, ribotyping, IS200 fingerprinting, and pulsedfield gel electrophoresis. J Clin Microbiol 37, 1687-1692.

Old, D. C., Chisholm, S. A., Crichton, P. B. \& Taylor, A. (2000). Grouping of Salmonella enterica serotype Montevideo strains by ribotyping and IS200 profiling. Epidemiol Infect 124, 375-382.

Olsen, J. E., Skov, M. N., Threlfall, E. J. \& Brown, D. J. (1994). 
Clonal lines of Salmonella enterica serotype Enteritidis documented by IS200-, ribo-, pulsed-field gel electrophoresis and RFLP typing. $J$ Med Microbiol 40, 15-22.

Olsen, J. E., Skov, M. N., Angen, Ø., Threlfall, E. J. \& Bisgaard, M. (1997). Genomic relationships between selected phage types of Salmonella enterica subsp. enterica serotype typhimurium defined by ribotyping, IS200 typing and PFGE. Microbiology 143, 1471-1479.

Osaka, K., Inouye, S., Okabe, N. \& 7 other authors (1999). Electronic network for monitoring travellers' diarrhoea and detection of an outbreak caused by Salmonella enteritidis among overseas travellers. Epidemiol Infect 123, 431-436.

Page, R. D. (1996). TreeView: an application to display phylogenetic trees on personal computers. Comput Appl Biosci 12, 357-358.

Pelkonen, S., Romppanen, E. L., Siitonen, A. \& Pelkonen, J. (1994). Differentiation of Salmonella serovar Infantis isolates from human and animal sources by fingerprinting IS200 and 16S rrn loci. J Clin Microbiol 32, 2128-2133.

Powell, N. G., Threlfall, E. J., Chart, H. \& Rowe, B. (1994). Subdivision of Salmonella enteritidis PT 4 by pulsed field gel electrophoresis: potential for epidemiological surveillance. FEMS Microbiol Lett 119, 193-198.

Reeves, M. W., Evins, G. M., Heiba, A. A., Plikaytis, B. D. \& Farmer, J. J., III (1989). Clonal nature of Salmonella typhi and its genetic relatedness to other salmonellae as shown by multilocus enzyme electrophoresis, and proposal of Salmonella bongori comb. nov. J Clin Microbiol 27, 313-320.

Ridley, A. M., Punia, P., Ward, L. R., Rowe, B. \& Threlfall, E. J. (1996). Plasmid characterization and pulsed-field electrophoretic analysis demonstrate that ampicillin-resistant strains of Salmonella enteritidis phage type 6a are derived from Salm. enteritidis phage type 4. J Appl Bacteriol 81, 613-618.

Ridley, A. M, Threlfall, E. J. \& Rowe, B. (1998). Genotypic characterization of Salmonella enteritidis phage types by plasmid analysis, ribotyping, and pulsed-field gel electrophoresis. J Clin Microbiol 36, 2314-2321.

Rowe, B. \& Hall, M. L. M. (1989). Kauffmann-White Scheme 1989. London: Laboratory of Enteric Pathogens, Central Public Health Laboratory.

Saitou, N. \& Nei, M. (1987). The neighbor-joining method: a new method for reconstructing phylogenetic trees. Mol Biol Evol 4, 406-425. Sanchez, G., Restrepo, S., Duque, M. C., Fregene, M., Bonierbale, M. \& Verdier, V. (1999). AFLP assessment of genetic variability in cassava accessions (Manihot esculenta) resistant and susceptible to the cassava bacterial blight (CBB). Genome 42, 163-172.

Selander, R. K. \& Musser, J. M. (1990). Population genetics of bacterial pathogenesis. In The Bacteria, a Treatise on Structure and Function, vol. XI, Molecular Basis of Bacterial Pathogenesis, pp. 11-31. Edited by B. H. Iglewiski \& V. L. Clark. New York: Academic Press.
Selander, R. K. \& Nelson, K. (1996). Evolutionary genetics of Salmonella enterica. In Escherichia coli and Salmonella: Cellular and Molecular Biology, vol. 2, pp. 2691-2707. Edited by F. C. Neidhardt. Washington, DC: American Society for Microbiology.

Selander, R. K., Caugant, D. A., Ochman, H., Musser, J. M., Gilmour, M. N. \& Whittam, T. S. (1986). Methods of multilocus enzyme electrophoresis for bacterial population genetics and systematics. Appl Environ Microbiol 51, 873-884.

Selander, R. K., Beltran, P., Smith, N. H., Barker, R. M., Crichton, P. B., Old, D. C., Musser, J. M. \& Whittam, T. S. (1990a). Genetic population structure, clonal phylogeny, and pathogenicity of Salmonella paratyphi $\mathbf{B}$. Infect Immun 58, 1891-1901.

Selander, R. K., Beltran, P., Smith, N. H. \& 7 other authors (1990b). Evolutionary genetic relationships of clones of Salmonella serovars that cause human typhoid and other enteric fevers. Infect Immun 58, 2262-2275.

Selander, R. K., Smith, N. H., Li, J., Beltran, P., Ferris, K. E., Kopecko, D. J. \& Rubin, F. A. (1992). Molecular evolutionary genetics of cattle-adapted serovar Salmonella dublin. J Bacteriol 174, 3587-3592. Stanley, J., Jones, C. S. \& Threlfall, E. J. (1991). Evolutionary lines among Salmonella enteritidis phage types are identified by insertion sequence IS200 distribution. FEMS Microbiol Lett 82, 83-89.

Stanley, J., Burnens, A., Powell, N., Chowdry, N. \& Jones, C. (1992). The insertion sequence IS200 fingerprints chromosomal genotypes and epidemiological relationships in Salmonella heidelberg. J Gen Microbiol 138, 2329-2336.

Swofford, D. L. (2000). PAUP*. Phylogenetic Analysis Using Parsimony (*and Other Methods). Sunderland, MA: Sinauer Associates.

Thong, K. L., Ngeow, Y. F., Altwegg, M., Navaratnam, P. \& Pang, T. (1995). Molecular analysis of Salmonella enteritidis by pulsed-field gel electrophoresis and ribotyping. J Clin Microbiol 33, 1070-1074.

Threlfall, E. J., Rowe, B. \& Ward, L. R. (1989). Subdivision of Salmonella enteritidis phage types by plasmid profile typing. Epidemiol Infect 102, 459-465.

Threlfall, E. J., Hampton, M. D., Chart, H. \& Rowe, B. (1994). Use of plasmid profile typing for surveillance of Salmonella enteritidis phage type 4 from humans, poultry and eggs. Epidemiol Infect 112, 25-31.

Uzzau, S., Brown, D. J., Wallis, T., Rubino, S., Leori, G., Bernard, S., Casadesus, J., Platt, D. J. \& Olsen, J. E. (2000). Host adapted serotypes of Salmonella enterica. Epidemiol Infect 125, 229-255.

Wang, F. S., Whittam, T. S. \& Selander, R. K. (1997). Evolutionary genetics of the isocitrate dehydrogenase gene (icd) in Escherichia coli and Salmonella enterica. J Bacteriol 179, 6551-6559.

Ward, L. R., de Sa, J. D. H. \& Rowe, B. (1987). A phage-typing scheme for Salmonella enteritidis. Epidemiol Infect 99, 291-294.

Weide-Botjes, M., Kobe, B. \& Schwarz, S. (1998). Inter- and intraphage type differentiation of Salmonella enterica subsp. enterica serovar enteritidis isolates using molecular typing methods. Zentbl Bakteriol 288, 181-193. 